Beyond doubt in a dangerous world: The effect of existential threats on the expression of certainty in societal discourse

\footnotetext{
Almog Simchon ${ }^{1 *}$, Chaya Turkin ${ }^{1}$, Tal Svoray ${ }^{1,2}$, Itai Kloog ${ }^{2}$, Michael Dorman ${ }^{2}$, and Michael Gilead $^{1 *}$

${ }^{1}$ Department of Psychology, Ben-Gurion University of the Negev, Israel, ${ }^{2}$ Department of Geography and Environmental Development, Ben-Gurion University of the Negev, Israel *almogsi@post.bgu.ac.il; mgilead@bgu.ac.il
}

The following is the accepted, pre-print manuscript of an article being published in an upcoming issue of Journal of Experimental Social Psychology: Special Issue on the interplay between individual and collective efforts 


\begin{abstract}
What happens when entire populations are exposed to news of impending existential threats? In the current study, we address this question by investigating the association between existential threats and the certitude of societal discourse. According to appraisal theory, threats give rise to anxiety and perceptions of uncertainty; as such, it predicts that exposure to life-threatening events will increase expressions of uncertainty. An alternative possibility is that people will respond to threats by utilizing psychological compensation mechanisms that will give rise to greater expressions of certainty. Across two studies, we measured linguistic certainty in more than 3.2 million tweets, covering different psychological contexts: (i) the 15 major terrorist and school shooting events that took place between 2016-2018; (ii) the COVID-19 pandemic. Consistent with the idea of compensatory processing, the results show that levels of expressed certainty increased following intentional and natural existential threats. We discuss the implications of our findings to theories of psychological compensation and to our understanding of collective response in the age of global threats.
\end{abstract}

Keywords: big data, Terror Management Theory, emotion, social discourse, motivated reasoning 


\section{Beyond doubt in a dangerous world: The effect of existential threats on the expression of certainty in societal discourse}

It is hard to think of a more disruptive, unsettling global health crisis than the 2020 coronavirus (COVID-19) pandemic. The novel coronavirus which rapidly swept through the earth was unfamiliar to health practitioners and the scientific community, giving rise to much uncertainty (e.g., How deadly is it? What will be the trajectory of the epidemic? Is the vaccine safe?). Despite these many unknowns, it could be argued that the public discourse concerning the nature of the disease did not involve excessive nuance or epistemic humility. In fact, in many cases, discussions of the proper response to the disease were characterized by surprising certitude and confidence.

An open and free society relies on the exchange of ideas, and the ability of individuals to reason with complexity and nuance - and to (sometimes) change their minds. As such, an overly dogmatic (Rokeach, 1954) societal discourse - wherein conversants are incapable of moderation and self-doubt — can undermine liberal democracy (van Prooijen \& Krouwel, 2017) and its ability to effectively respond to challenges. Thus, as the case of COVID-19 may highlight, it is important to understand what affects the dogmatism of discourse in a given society. In the current paper we explore one potential reason for humans' tendency for certitude — our inherent fear of death.

\section{What is the effect of existential threats on dogmatism?}

In today's world of 24-hours news and social media, entire populations are constantly exposed to threatening images on their smartphone and television screens-depicting an unpredictable, dangerous world: in England, a suicide bomber takes the lives of parents and 
children exiting a pop-star concert; in Florida, a teenager visits his former high school to sow carnage; in New York, mass graves are being dug for the casualties of the COVID-19 pandemic.

It could be argued that in the past, our world wasn't safer or more predictable (Pinker, 2011), only that reminders of our mortality were less prevalent and vivid. For example, the 1968 "Hong Kong Flu" took the lives of an estimated 1 million people across the world, and 100,000 within the United States (Kilbourne, 2006) — yet remained somewhat of a footnote in the history books (e.g., Hays, 2005). In the hyperconnected reality of 2020, the emergence of a pandemic of somewhat similar magnitude has become what seems to be an era-defining event.

The heightened media exposure to the global and domestic crises has many adaptive benefits. For example, in the case of the coronavirus pandemic, the appreciation of the fatal risks posed by the illness prompted unprecedented precautionary steps all over the world, successfully minimizing the loss of lives. At the same time, it is likely that a heightened sense of a menacing world has non-negligible psychological and social consequences. In light of this, in the current manuscript we ask - what happens when entire populations are exposed to news of impending existential threats?

The exposure to media depictions of a dangerous world confronts individuals with existential uncertainties - How can we protect ourselves? Is it safe to send our children to school? According to appraisal theory (Lazarus, 1991) perceptions of uncertainty are a central dimension in the emergence of anxiety; indeed, research has convincingly shown that feelings of anxiety often generate a subsequent sense of uncertainty in individuals (Raghunathan \& Pham, 1999). In light of this, a straightforward answer to the question posed above is that the increase in anxiety 
following exposure to news depictions of a dangerous world will cause individuals to experience and express increased levels of uncertainty.

However, an alternative possibility is that individuals may respond to "ripples of uncertainty" (Maguen et al., 2008) by utilizing psychological compensation mechanisms that strive to restore their psychological equilibrium. The idea that existential threats would be associated with a greater (rather than diminished) sense of certainty is consistent with several theories that describe such psychological compensation mechanisms, e.g., the Meaning Maintenance Model (Heine et al., 2006), Uncertainty Management Model (UMM; van den Bos et al., 2005; van den Bos \& Lind, 2002), and Terror Management Theory (Greenberg et al., 1986) (see General Process Model of Threat and Defense for a comprehensive discussion; Jonas et al., 2014).

For example, TMT (Greenberg et al., 1986), suggests that when confronted with their mortality, people will find comfort in the eternal existence of their culture and values; thus, in response to existential threats, they will hold on firmly to their existing belief system and the sense of meaning it affords. Within TMT, research has shown that people respond to a reminder of their mortality by exhibiting more extreme attitudes (Canetti-Nisim et al., 2009) and expressing more confidence in their opinions (Jong et al., 2012).

Bolstered expressions of certainty may be also anticipated by some appraisal theories. For example, Appraisal Tendency Framework (ATF) predicts that existential threats can lead to enhanced certainty if they predominantly give rise to feelings of anger. Specifically, ATF (and appraisal theory more generally, e.g., Ellsworth \& Scherer, 2003; Smith \& Ellsworth, 1985) posits that whereas perceptions of anxiety are associated with lower levels of certainty, 
perceptions of anger are associated with increased certainty (Lerner et al., 2007; Lerner \& Keltner, 2000; Lerner \& Keltner, 2001). Supporting this idea, research has shown that existential dangers that are caused by hostile human agents often predominantly evoke anger (rather than anxiety) — and as such, give rise to an increased (rather than decreased) sense of certainty and control (Motro \& Sullivan, 2017). In addition, participants reported feeling greater uncertainty concerning unsettling events after being induced to feel fear vs. anger (Lerner \& Keltner, 2001).

Thus, whereas some research suggests that the effects of anxiety induced by exposure to reports of existential dangers would be in increased expression of uncertainty, it is also possible that such threats will lead to increased expression of certainty - either via the workings of psychological compensation mechanisms or via activation of appraisal tendencies associated with feelings of anger.

\section{The current research}

In the present study, we sought to examine how exposure to depictions of a dangerous world affect societal discourse, by utilizing the newly-available capacity to observe the real-time reaction of the public to existential threats, as it is reflected in social media discourse (for a similar approach see Dore et al., 2015; Fan et al., 2019). One of the main advantages of this type of research is the option to conduct behavioral studies at an unprecedented scale; when sample sizes grow from hundreds to hundreds-of-thousands, questions of replicability (Pashler \& Wagenmakers, 2012) and generalizability (Yarkoni, 2020) are of lesser concern.

Another advantage of big-data research lies in its potential ecological validity. While the classic experimental design (i.e., random assignment to groups by an experimenter) is rightfully considered the gold standard for causal inference, the practical and ethical limits of random 
assignment studies can sometimes restrict the ecological validity of experimental models (Leatherdale, 2019). In the current study we leveraged a "natural experiment" design, in which the explanatory variable is highly exogenous and extremely unlikely to be caused by the outcome variable. Such ecologically-valid natural experiments are underused in psychological research, despite the fact that they can often support relatively strong causal inferences (Grosz et al., 2020).

Investigating online discourse is an opportunity to explore how large-scale events are construed by-, and affect individuals — as reflected in their linguistic behavior on social media. Moreover, social media discourse, in turn, can be seen as a form of collective "action" that subsequently shapes the minds of those who are exposed to it. As such, big-data analyses can elucidate both sides of the interplay between individual and collective efforts in the age of global threats, discussed in this special issue - in that it allows us to examine how collective threats influence the individual, and how their psychological reactions, in turn, are manifested as a distributed collective response.

In Study 1, we explored the levels of expressed certainty on the social network Twitter following the major terror attacks and mass shootings that occurred in the United States and the United Kingdom between the years of 2016-2018; in Study 2, we examined the effect of the COVID-19 pandemic.

\section{Study 1 - the effect of mass murder events on linguistic certainty}

Individuals in the western world describe the threat of terrorism to be one of the greatest concerns in their daily lives (Brück \& Müller, 2010). As such, it is becoming increasingly important to gain a better understanding of the psychological reactions to media reports of mass 
murder events. Research examining the response to terrorist events has shown that exposure to media coverage of terrorism increase reports of anxiety (Slone \& Shoshani, 2006); similarly, research that examined online discourse following mass shootings has shown that individuals respond to such events by expressing higher levels of anxiety (Dore et al., 2015). In light of this, in Study 1 we sought to investigate how such depictions of a dangerous world affect the certainty-expressions in online social discourse.

Appraisal Tendency Framework (ATF) would predict that existential threats should cause people to experience and express lower levels of certainty (Lerner et al., 2007; Lerner \& Keltner, 2000; Lerner \& Keltner, 2001); the compensatory mechanisms approach would predict that when coping with existential anxiety, people will hold on to their worldviews and therefore express greater certainty in their language (Greenberg et al., 1986; Heine et al., 2006; van den Bos et al., 2005).

While the ATF and compensatory process theories (i.e., TMT, UMM, MMM) generate divergent predictions, we should note that the current study was not designed in order to try and refute or support these frameworks. Rather, the studies reported herein are of an exploratory nature; in the terminology of Schindler et al. (2021), these studies are an example of the "discovery mode" of the scientific enterprise.

\section{Method}

\section{Data collection}

Main database. Since October 2016, our lab has been continuously collecting Twitter data. We regularly sample tweets from all 50 states in the United States, including the District of Columbia. Tweets were sampled on an hourly basis from two regions in each state. One region 
was centered at the most populated city in the state, and the other region was centered at the state's approximate geographic center. The radii of each of the two regions were determined such that they covered the maximal area without crossing state borders, and minimizing overlap with each other. These sampling decisions are not specific to the current study; they reflect our $a$ priori criterion for twitter data collection.

Additional data. For events prior to October 2016, we used a different repository, smaller in size, collected by some of the authors. For events outside the United States, tweets were gathered by targeting a radius of a few hundred kilometers from the attack's location. In the case of event \#8 (the Manchester attack), we were too late to collect control days data from the Twitter API, therefore, we bought the data from a third-party, a company that offered historical Twitter data, Texifter's Sifter service.

Event Selection. In order to target possible terrorist/mass shooting events available in our database, we relied on the Johnston's Archive ${ }^{1}$. The events were: (1) Orlando, FL, June 2016; (2) Dallas, TX, July 2016; (3) Bristol, TN, July 2016; (4) Baton Rouge, LA, July 2016; (5) Columbus, OH, November 2016; (6) Fort Lauderdale, FL, January 2017; (7) Fresno, CA, April 2017; (8) Manchester, UK, May 2017; (9) London, UK, June 2017; (10) London, UK, September 2017; (11) Las Vegas, NV, October 2017; (12) NYC, NY, October 2017; (13) Sutherland Springs, TX, November 2017; (14) Parkland, FL, February 2018; (15) Pittsburgh, PA, October 2018. For each site of terror attack/mass shooting, we analyzed the data in that state where the attack had occurred. Our analysis relied on a time period beginning with the time of the event as

\footnotetext{
${ }^{1}$ http://www.johnstonsarchive.net/terrorism/wrjp255a.html
} 
reported in the media, until the end of the same day; control segments were exactly one week prior to the terror incident.

\section{Procedure}

Prior to May 2018, access to the Twitter Application Programming Interface (API) and data collection was made through the twitteR (Gentry, 2012) package. Since May 2018, tweets were collected using the Python Twitter Tools package (Verdone, 2014). All retweets and thirdparty posts were removed (restricting the source of the post to Twitter or Facebook, as some users tend to repost their Facebook posts as tweets). All the text was cleaned from links, mention-tags and emoticons.

\section{Measures}

Tweets were analyzed using Linguistic Inquiry and Word Count (LIWC; Pennebaker et al., 2015), a computerized text analysis program. LIWC is a natural language processing tool that measures the relative occurrence of words from an embedded dictionary for a specific text input. For example, given the string "I am sad", LIWC will return a sadness score of 33.33 (i.e. $1 / 3$ of the tweet consists of words that are found in the sadness dimension), and a 33.33 score of personal pronouns (i.e., $1 / 3$ of the tweet consists of the personal pronoun $I$ ). LIWC contains dozens of linguistic and psychological dictionaries that have been extensively investigated and validated over the years (e.g., Kahn et al., 2007; Pennebaker et al., 1997).

Our analysis looked at several relevant LIWC dimensions (tentativeness, anxiety, anger, and negative emotion), however, our main variable of interest was certainty - a dictionary that has been compiled in order to gauge the degree of resoluteness and inflexibility expressed in texts (Cohen, 2012). 
Many studies have provided examples of the criterion validity of the LIWC certainty dictionary (Pezzuti et al., 2021; Shields et al., 2013; Sterling et al., 2020). For example, Fast and Horvitz (Fast \& Horvitz, 2016) asked participants to rate comments posted on the social media platform Reddit for their level of dogmatism - that is, the degree to which they perceive the author as unwilling to discuss and consider other viewpoints. Perceived dogmatism positively correlated with LIWC's certainty, and negatively correlated with LIWC's tentativeness dictionary. Moreover, research also provided evidence for the relation between certainty-related language — as it is measured by LIWC — and consequential real-world behaviors; for example, the degree of linguistic certainty in the rulings of the US supreme court is associated with the degree of compliance exhibited by lower courts (Corley \& Wedeking, 2014).

\section{Preprocessing and statistical modeling}

Our sample size consisted of 2,547,199 observations. We aggregated these observations by user, event, and condition (mass casualty event or control). Because of the large number of observations, conducting a mixed-model analysis required immense computational power and was not feasible. Therefore, we removed all dependencies in the data (i.e., users who tweeted both before an attack and after the attack, resulting in removing $20 \%$ of the data). The final sample size of two events (Bristol, TN, July 2016; Baton Rouge, LA, July 2016) consisted of less than 50 observations in each condition, and therefore were removed from the analysis (this decision did not impact the findings, see online supplementary for the full analysis). Our final sample size consisted of 482,289 unique data points.

We then converted the aggregated LIWC scores to raw count data by multiplying by the word count and rounding to the nearest integer. This practice was motivated by two issues: (a) using linear-models for zero-inflated data is a significant divergence from the model assumptions 
and (b) simulation studies within our lab show greater statistical power for modeling word frequencies in the appropriate count-models (e.g., Poisson, negative-binomial) vs. the current practice (i.e., linear models). We fitted a negative binomial count model using the `MASS` R package (Ripley et al., 2013).

\section{Results}

In Table 1 we present a summary table of LIWC measures by time and event. In the supplementary repository we provide all LIWC dimensions. Figure 1 depicts the same summary statistics for the certainty dimension. 
Table 1. Summary table of LIWC dimensions by time and event. Means and standard deviations in parenthesis. Mean value represents average LIWC scores of all tweets by condition (Before/After). Scores are the counts of a specific dictionary in a tweet divided by tweet length.

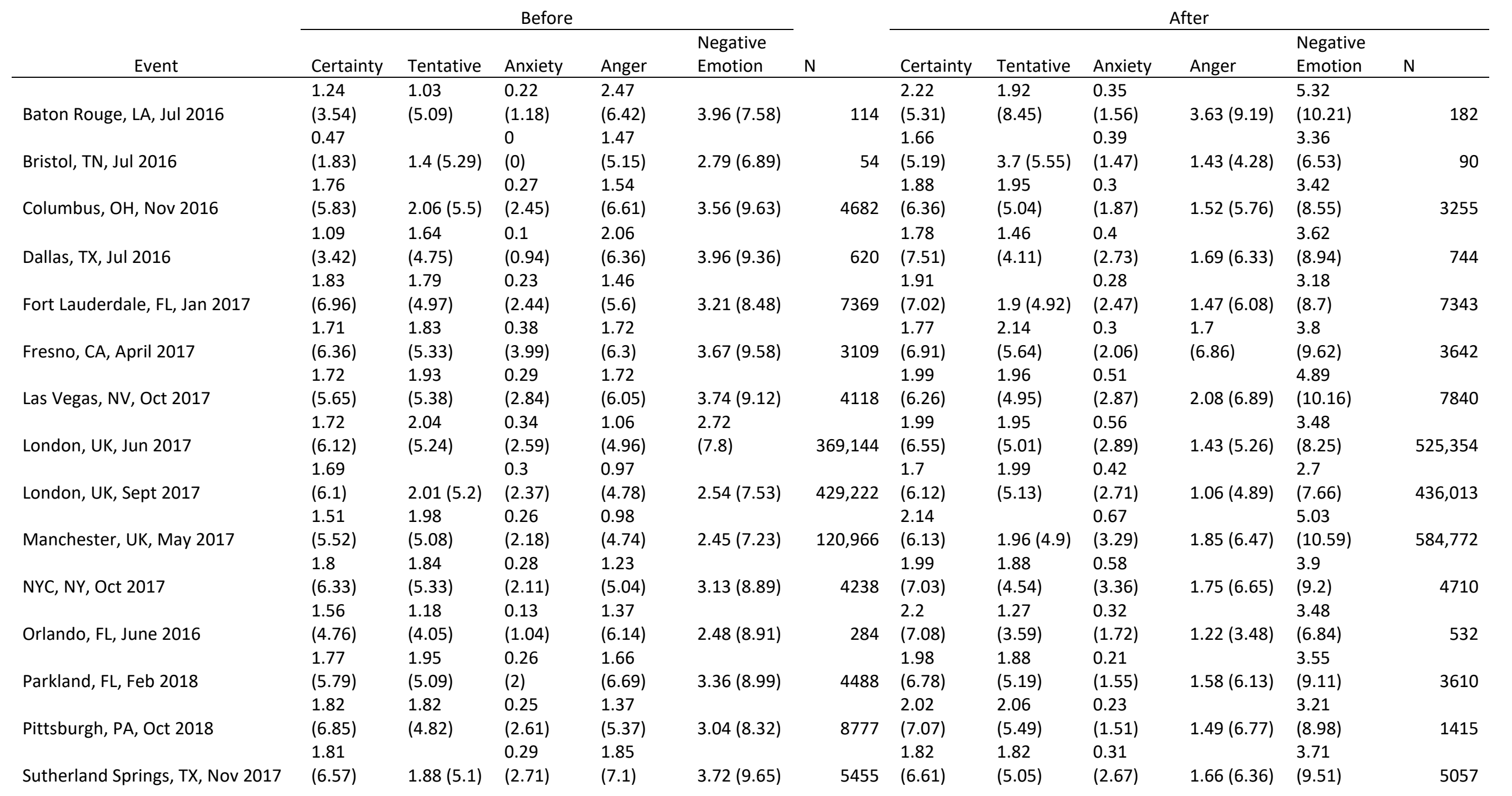




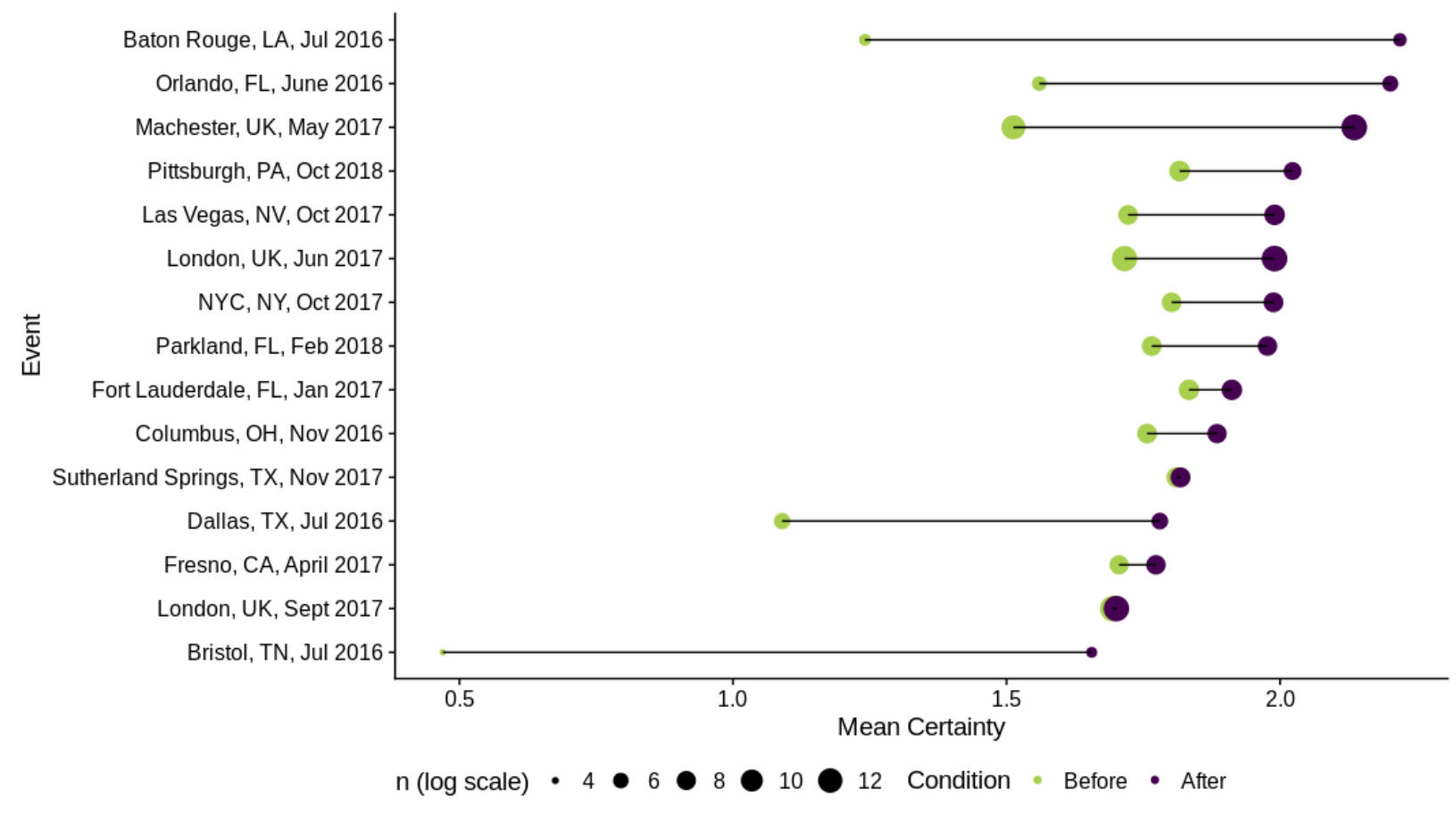

Figure 1. Mean certainty by event and condition. light green represents the time before the event, dark purple represents the time after the event. The size of the point denotes the sample size on the log scale.

We fitted a model that predicted the number of certainty-related words by the quasiexperimental condition (one week before the attack/right after the attack); word count and event were held as covariates. The results show that following a terror event, there was a $21 \%$ increase in certainty words $(\mathrm{IRR}=1.21, p<.001,95 \% \mathrm{CI}=1.19,1.23$; Table $\mathrm{S} 1)$. We fitted another model, wherein instead of controlling for events, we controlled for the number of fatalities (these could not be fitted together since the number of fatalities does not vary within events). Here too, following a mass violence event, there was a $24 \%$ increase in certainty words (IRR $=1.24, p<$ $.001,95 \% \mathrm{CI}=1.22,1.26$; see Table 2).

We followed the same procedure in predicting tentative words. The results show that after a terror event there was a $5 \%$ decrease in tentative words $(\mathrm{IRR}=0.95, p<.001,95 \% \mathrm{CI}=$ 
$0.94,0.96$; Table S2). In the same manner, we fitted a model wherein the number of fatalities is controlled for instead of events, resulting in a 7\% decrease in tentative words (IRR $=0.93, p<$ $.001,95 \% \mathrm{CI}=0.91,0.94$; Table S3). Thus, both models provide a conceptual mirror-image of the certainty analysis.

Table 2. Summary of Negative Binomial Regression Analysis for variables predicting certaintyrelated word count including death toll as covariate.

\begin{tabular}{lccc}
\hline $\begin{array}{l}\text { Predictors } \\
\text { Incidence Rate } \\
\text { Ratios }\end{array}$ & $C I$ & $p$ \\
\hline Time (Before\After) & 1.24 & $1.22-1.26$ & $<\mathbf{0 . 0 0 1}$ \\
Death Toll & 1.01 & $1.00-1.01$ & $<\mathbf{0 . 0 0 1}$ \\
Word Count & 1.09 & $1.09-1.09$ & $<\mathbf{0 . 0 0 1}$ \\
\hline Observations & & & \\
\hline
\end{tabular}

To better understand what kind of linguistic input drives the effect, we extracted word frequencies of certainty-related language, and conducted a Differential Language Analysis (e.g., Schwartz et al., 2013). We created two proportion tables for before and after events and calculated difference scores. Figure 2 visualizes this analysis in the form of a word cloud. The figure shows that the biggest contributors to the effect were the words all and everyone in postevent tweets. Such words are considered universal quantifiers that generate "blanket statements" that do not leave room for much nuance or moderation, and are therefore considered as reflecting 
lack of tentativeness (in comparison to quantifiers such as "many", "some", "several"). However, the words "everyone" and "all" can have meanings that reflect social unity and cohesion—which are theoretically related to the need for greater epistemic certainty (Garcia \& Rimé, 2019; Hardin \& Higgins, 1996; Kopietz et al., 2010)—yet can be considered as distinct psychological constructs.

Therefore, we conducted an additional analysis wherein all tweets containing the words all and everyone were excluded from the analysis. Results show that even when these universal quantifiers are omitted from the analysis, mass murder events are still associated with a $11 \%$ increase in certainty words $(\mathrm{IRR}=1.11, p<.001,95 \% \mathrm{CI}=1.09,1.13$; see Table $\mathrm{S} 4$ in SM)

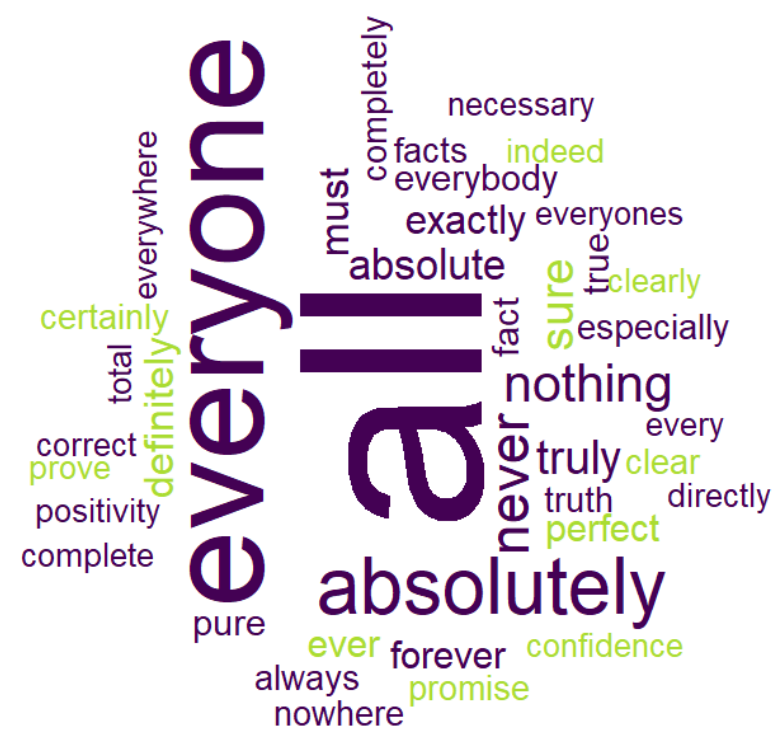

Figure 2. Word cloud of certainty-related language. The size of the word represents its unique association strength with either before an event (light green) or after an event (dark purple). Showing 40 most impactful words.

To what extent is the increase in certainty driven by anxiety and anger? According to the Appraisal Tendency Framework (ATF), the increase in certainty related language 
following mass shooting events should be predominantly driven by anger. Any increase in anxiety following such events should suppress the effect of the anger-inducing event and expressions of certainty.

To test this prediction, we examined whether anxiety or anger mediated the effects of existential threat on expression of certainty. Like certainty, levels of anxiety and anger were measured via LIWC. We conducted a causal mediation analysis using the `mediation` R package (Tingley et al., 2014), applying a Poisson generalized linear model ${ }^{2}$, and a bootstrap method with 10,000 iterations.

When considering anxiety as a mediator, the effect of the direct path was statistically significant $($ mean direct effect $=0.03,95 \% \mathrm{CI}$ of bootstrapped samples $=[0.03,0.04], p<.001)$, and the mediation was significant (proportion of the effect mediated by anxiety was $1.4 \%, 95 \%$ $\mathrm{CI}=[0.01,0.02], p<.001)$. When considering anger as a mediator, again the direct path was significant $($ mean direct effect $=0.03,95 \% \mathrm{CI}$ of bootstrapped samples $=[0.03,0.04], p<.001)$, however, the proportion of the effect mediated by anger was only $-0.03 \%$ and the mediation did not attain statistical significance $(95 \% \mathrm{CI}=[-0.003,0.008], p=.078)$. The negative sign of the mediated effect suggests, if anything, anger is a suppressor, rather than mediator. Detailed mediation results are shown in Table S5 in SM. Thus, the results of the mediation analysis indicate that anxiety had a larger role in mediating the effect than anger.

\section{To what extent is the increase in certainty driven by general negative affect?}

\footnotetext{
${ }^{2}$ A Poisson model is a special case of the Negative Binomial (NB) model. Ideally, we would have fitted a NB model, which does not include an assumption about mean and variance equality. However, we could not find a stable implementation of a NB mediation analysis. A Poisson mediation model makes the next best alternative and is unlikely to significantly differ from a NB model.
} 
Experimental research that directly investigates the premises of threat management theories such as TMT often contrasts the effects of an existential anxiety manipulation with those of a control condition that elicits general negative affect (e.g., recollection of a visit to a dentist; Jonas et al., 2002; Pyszczynski et al., 2006). Such a control helps rule out the possibility that the observed effect is simply attributable to general affective processes, rather than existential anxiety. While experimental control is impossible in our quasi-experimental design, we sought to examine whether the effects of the existential threats on certainty related language are primarily driven by general negative affect, or whether it is indeed the case that anxiety has a unique role in giving rise to increased certainty.

To address this possibility, we conducted an additional analysis where we included within the model a covariate of general negative affect, as measured by LIWC. First, we fitted a negative binomial model to predict negative emotion, and saw that indeed, negative affect was higher following a terror attack $(\mathrm{IRR}=1.50, p<.001,95 \% \mathrm{CI}=1.48,1.52$; see Table S6 in SM). Importantly, the results showed that even after controlling for general negative affect, there was still a $19 \%$ increase in certainty words following exposure to mass violence events (IRR = 1.19, $p<.001,95 \% \mathrm{CI}=1.17,1.21$; see Table S7 in SM). This trend holds even after excluding all the tweets containing the words all and everyone from the analysis (IRR $=1.10, p<.001$, $95 \% \mathrm{CI}=1.07,1.12 ;$ see Table S8 in SM).

\section{Study 1 Discussion}

The results of Study 1 showed that senseless acts of mass murder result in increased use of language that reflects anxiety and increased use of language that reflects certainty of one's assertions. Such findings are consistent with the workings of psychological compensation mechanisms that seek to restore psychological equilibrium in light of existential threat. 
Furthermore, as we noted, the results of the mediation analysis are not consistent with an Appraisal Tendency Framework interpretation, according to which increased anxiety should result in subsequent decreases in certainty, whereas increased anger should increase certainty.

However, the ability to draw causal mechanistic conclusions based on the analysis of covariances in mediation analyses is quite limited (Bullock et al., 2010). Thus, to rule out an ATF interpretation of the findings, it is informative to examine cases wherein the relation between existential anxiety and certainty is unconfounded with feelings of anger, such as those likely elicited by vicious, murderous attacks that take the lives of innocent people.

Feelings of anger prompt a "fight" (rather than "flight") response — and thereby enhance chances of victory in conflict situations with conspecifics (Sell et al., 2009; Tamir et al., 2008); as such, anger involves the appraisal of the harms being purposely intentionally afflicted by human agents (Betancourt \& Blair, 1992; Russell \& Giner-Sorolla, 2011). Research has shown that whereas threats elicited by natural disasters elicit fear and do not increase people's sense of certainty, threats elicited by human agents indeed give rise to increased anger — and subsequently to an increased sense of control and certainty (Motro \& Sullivan, 2017). In light of this, in Study 2 we sought to examine whether threats that have natural (rather than an intentional) origin may also increase linguistic certainty.

\section{Study 2 - effects of a deadly pandemic on linguistic certainty}

The coronavirus disease (COVID-19) was first detected in Wuhan, China in December 2019 and within a few months had spread to most of the world. As of June 2021, the novel coronavirus had affected more than 181 million people and had taken a toll of almost 4 million lives (Dong et al., 2020). Governments around the world took aggressive prevention methods, 
including social distancing and lockdowns unprecedented in scope. The threat the pandemic poses and its immediate severe ramifications and disruption to the vast majority of the world population, have made it the top news story of recent years: billions of people around the world were exposed on a regular basis to news reports of the spread of the virus and its horrendous death toll.

Similar to acts of mass murder, the coronavirus sowed great emotional turmoil among people, which resulted in immediate mental health outcomes (Wang et al., 2020). However, unlike such acts of intentional harm, the carnage sowed by the virus was not the result of human malevolence. Therefore, we were interested in the effects this kind of natural threat has on expressions of certainty. Past research has shown that natural threats such as the COVID-19 pandemic and global warming are perceived as posing an existential threat (Fritsche et al., 2012; Pyszczynski et al., 2021). We reasoned that if we find that expressions of certainty decrease with rising concern over a natural disaster, it would support an ATF interpretation of the finding of Study 1 . However, if we see an increase in certainty expression, this would be more consistent with the workings of compensation mechanisms.

\section{Method}

Following the design rationale of Study 1, we analyzed Twitter discourse in the state most affected by the threatening event, which, in the case of the COVID-19 pandemic, was New York state (which, at the time of the analysis was the state-level entity with the highest mortality rate in the world - one out of every 670 people in the state have died from the disease). 
Unlike Study 1, the COVID-19 pandemic was a gradually-evolving event, wherein levels of concern rose as fatalities rose. In light of this, instead of conducting a before-after analysis, we examined the correlation between certainty-related language and public concern.

In order to gauge the level of anxiety caused by the rapidly progressing pandemic we relied on coronavirus concern polls collected by the polling company Civiqs, an internet-based polling company. The polls represent daily net concern in the state of New York, i.e., answers to the question "How concerned are you about a coronavirus outbreak in your local area?". In the state of New York, Civiqs have collected 1,365 responses between February 25th 2020 and April 15 th 2020.

Our Twitter sampling approach remained as in Study 1. In the current study, we sampled 782,983 tweets that were posted from NY between February 25th and April 15th. All the messages were analyzed with LIWC, and aggregated by day, resulting in a total of 51 single dayaverages of LIWC scores. Unlike in Study 1, in Study 2 we did not use a generalized linear model of count data (i.e., Negative Binomial) because we were interested in the strength of the association between two continuous variables--and the daily aggregation had satisfied the assumption of residuals' normality, allowing us to fit a linear model.

\section{Results}

We correlated COVID-19 net concern in NY as reported by Civiqs poll, and NY-based Twitter expressions of certainty, anxiety, anger and negative emotion on a daily breakdown $(\mathrm{N}=$ 51), see Table 3. Daily changes in certainty-related language and NY net concern are shown in Fig. 3. Correlations with certainty $(r=.31, p=.02)$ and negative emotion $(r=.40, p=.003)$ 
remained significant, even after removing tweets that contained all and everyone. The full correlation matrix is reported in Table S9.

Table 3 . Means, standard deviations, and correlations of COVID-19 concern and Twitter expression of certainty, tentativeness, anxiety, anger and negative emotion between February 25th 2020 and April 15th 2020.

\begin{tabular}{|c|c|c|c|c|c|c|c|}
\hline Variable & $M$ & $S D$ & 1 & 2 & 3 & 4 & 5 \\
\hline 1. Net Concern & 47.31 & 26.45 & & & & & \\
\hline 2. Certainty & 1.86 & 0.09 & $\begin{array}{l}.48^{* *} \\
{[.24, .67]}\end{array}$ & & & & \\
\hline 3. Tentativeness & 1.88 & 0.08 & $\begin{array}{l}.16 \\
{[-.13, .41]}\end{array}$ & $\begin{array}{l}.17 \\
{[-.11, .43]}\end{array}$ & & & \\
\hline 4. Anxiety & 0.28 & 0.03 & $\begin{array}{l}-.02 \\
{[-.29, .26]}\end{array}$ & $\begin{array}{l}.22 \\
{[-.05, .47]}\end{array}$ & $\begin{array}{l}.47 * * \\
{[.23, .66]}\end{array}$ & & \\
\hline 5. Anger & 1.24 & 0.06 & $\begin{array}{l}-.23 \\
{[-.47, .05]}\end{array}$ & $\begin{array}{l}.05 \\
{[-.23, .32]}\end{array}$ & $\begin{array}{l}-.39 * * \\
{[-.60,-.12]}\end{array}$ & $\begin{array}{l}-.08 \\
{[-.35, .20]}\end{array}$ & \\
\hline $\begin{array}{l}\text { 6. Negative } \\
\text { Emotion }\end{array}$ & 2.97 & 0.11 & $\begin{array}{l}.35^{*} \\
{[.09, .57]}\end{array}$ & $\begin{array}{l}.39 * * \\
{[.13, .60]}\end{array}$ & $\begin{array}{l}-.22 \\
{[-.47, .06]}\end{array}$ & $\begin{array}{l}.16 \\
{[-.12, .42]}\end{array}$ & $\begin{array}{l}.63 * * \\
{[.43, .77]}\end{array}$ \\
\hline
\end{tabular}

Note. $M$ and $S D$ are used to represent mean and standard deviation, respectively. Values in square brackets indicate the $95 \%$ confidence interval for each correlation. * indicates $p<.05$. ** indicates $p<.01$. 


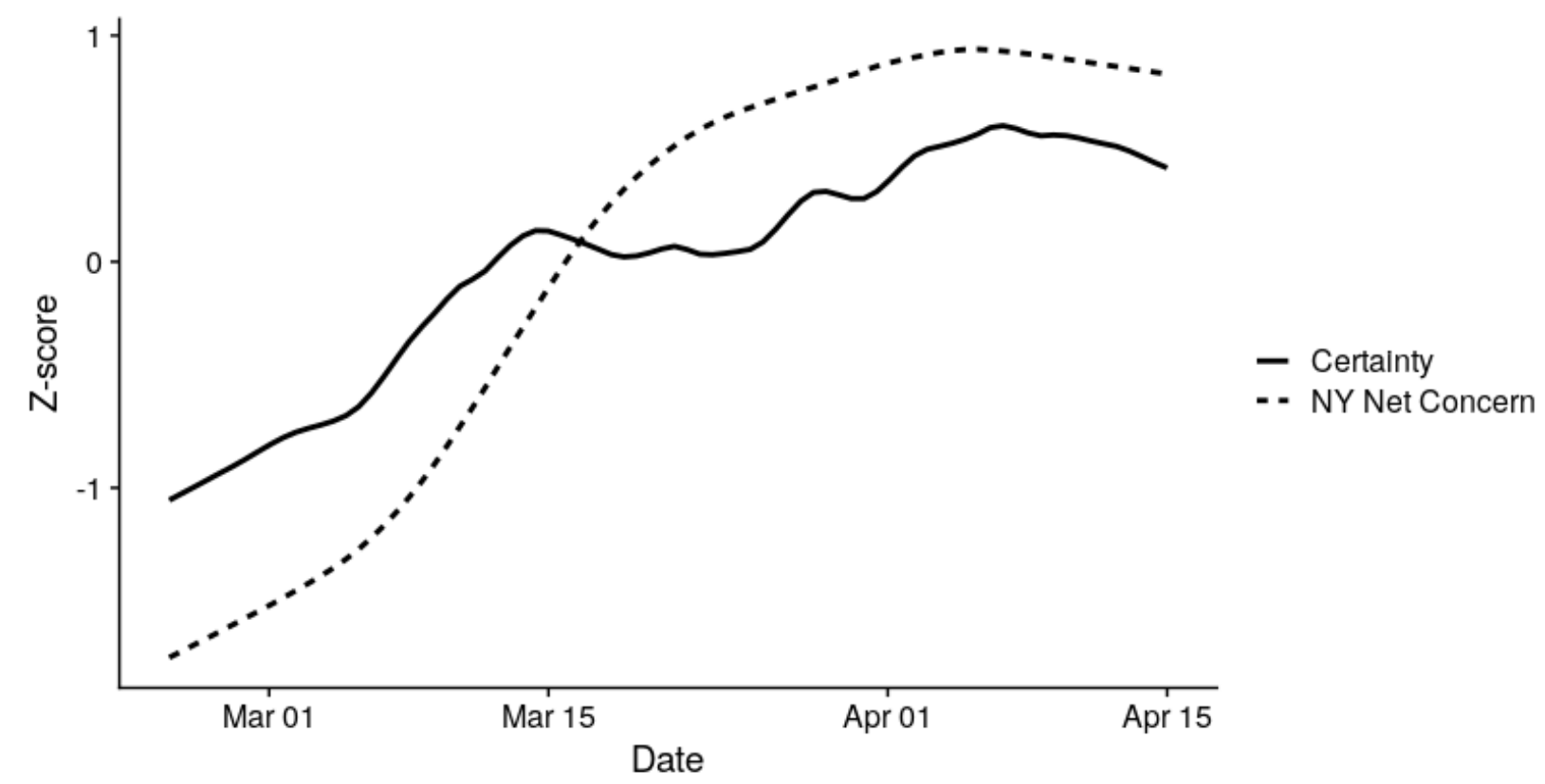

Fig 3. Certainty-related expressions on Twitter (solid line) and COVID-19 concern among New Yorkers (dashed line) between February 25th 2020 and April 15th 2020. Raw values were converted to standardized values. The functions have gone through a locally estimated scatterplot smoothing $(\operatorname{span}=0.33$, degree $=1)$.

We fitted a linear regression model, predicting certainty by COVID-19 concern and negative emotion $\left[R^{2}=.29, F(2,48)=9.67, p<.001\right]$. Even after controlling for negative emotion ( $\beta=0.25, p=.057)$, COVID-19 net concern remained a significant predictor $(\beta=0.39, p$ $<.01)$.

Like in Study 1, we were interested in exploring what kind of linguistic input drives the effect. For that purpose we analyzed the frequency of certainty-related words in each of the 51 days and applied a term frequency-inverse document frequency weighting scheme (tf-idf; a standard procedure used to assess word importance; Kennedy et al., 2021). We then correlated each word's daily tf-idf score with the corresponding daily COVID-19 net concern. Figure 3 
presents the certainty-related words that passed statistical significance at $p<.05$. Once again, absolutist words like everyone, must and total were positively associated with concern of existential threat. Interestingly, the word confidence was negatively associated with existential threat, which mimicked the results of Study 1. One of the major factors that drove the effect in Study 1 was the word all; similarly, in the current study it was positively correlated with concern over the existential threat, however it did not reach statistical significance $(r=0.25, p=.07)$.

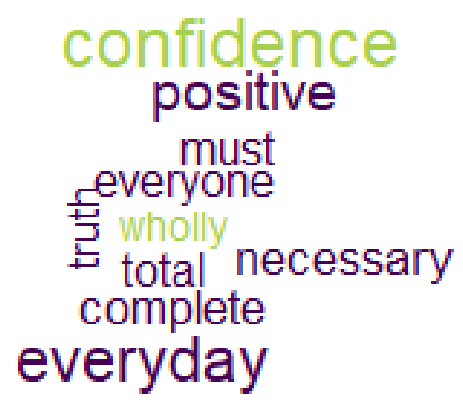

Figure 4. Word cloud of certainty-related language. The size of the word represents its unique association strength $(|r|)$, either positively associated with concern over COVID-19 (dark purple) or negatively (light green). Showing words that past significance testing at $p<.05$.

\section{Study 2 discussion}

The findings of Study 2 show that societal discourse increases in certitude following exposure to natural existential threats, contrary to predictions from ATF. Despite the fact that the COVID-19 pandemic was a natural disaster, it remains plausible that its progression was associated with increases in anger (e.g., anger at the government's delayed response to the pandemic; conspiracy theories regarding the origin of the virus). However, such a possibility is 
somewhat inconsistent with our results, wherein anger trended (albeit, not significantly) towards being negatively (rather than positively) correlated with feelings of concern. Thus, the findings are consistent with theories that posit that humans respond to existential threats by engaging compensatory mechanisms that serve to restore psychological equilibrium, such as TMT (Fritsche et al., 2012; Pyszczynski et al., 2021).

One possible limitation of the current study pertains to aggregate sample size $\left(\mathrm{N}_{\text {days }}=\right.$ 51), which in standard experimental settings would be considered on the lower end. However, this is not a typical design: we should think of days as participants, and messages as trials. For power consideration, researchers often tend to go with larger Ns as for most designs, increasing the number of trials is either impossible or unfeasible. With thousands of observations in each cell, aggregation minimizes the error and even with a small number of "participants", the results are quite robust (for the tradeoff between trials and participants see Brysbaert \& Stevens, 2018).

\section{General Discussion}

In the current research we observed that individuals respond to existential threats by using language that expresses increased certainty. These effects were evident in a sample that included millions of observations, and encompassed the major terrorist and mass shooting events that took place between 2016-2018 in the United States and the United Kingdom as well as the events of the 2020 COVID-19 pandemic.

\section{Which theories best explain the results?}

According to appraisal theory, feelings of anxiety are predominantly characterized by a sense of uncertainty. Indeed, research within ATF (Lerner \& Keltner, 2000) has shown that the experimental manipulation of anxiety gives rise to subsequent expressions of uncertainty (Lerner 
\& Keltner, 2001; Raghunathan \& Pham, 1999). The current findings, wherein language use following existential threats reflected increased (rather than decreased) expressions of certainty and anxiety are not readily explained by the appraisal framework. Instead, our findings are more consistent with the idea that individuals sometimes respond to threats by utilizing psychological compensation strategies.

For example, these findings are in line with (some of) the postulates of Terror Management Theory (Greenberg et al., 1986), which posits that when individuals come face to face with their mortality, they firmly hold on to their belief system with great certainty and conviction, and will thereby be comforted by a sense of "symbolic immortality" (Arndt et al., 1997).

Previous research within the TMT framework that has examined the effects of Mortality Salience (MS) on language use has shown that MS sometimes increases the use of language that reflects causation (Landau et al., 2009); it is associated with greater use of positive emotion words (Kashdan et al., 2014); and that MS boosts linguistic style mimicry with a conversation partner (Cox \& Kersten, 2016). Importantly, whereas most research into TMT has examined mortality salience in laboratory contexts, the current work provides evidence that comes from a highly ecological setting.

It is worth noting that the robustness of laboratory-based tests of TMT has been called into question (e.g., Schindler et al., 2021), especially in light of a recent large-scale failure to replicate findings from TMT research (Klein et al., 2019). Following this failed replication, the empirical status of TMT in itself was called into question by some (but see Chatard et al., 2020; Schindler et al., 2021, for a discussion of several severe limitations of the failed replications). Our current study was not intended to provide an evaluation of TMT; nonetheless, the results we 
obtained seem to us to be well explained by TMT. The findings may also suggest that future evaluations of the theory (and psychological science more broadly) could benefit from stepping outside of the (somewhat artificial) lab environment, and utilizing more ecologically-valid designs.

Another theoretical framework that can account for the current findings is the Meaning Maintenance Model (Heine et al., 2006). The Meaning Maintenance Model predicts that any event that substantially disrupts our view of the world as predictable would result in compensatory increases in sense of certainty, and treats existential threats as a specific case of this more general phenomenon. Future research could examine whether highly unpredictable events that do not entail any existential threats likewise give rise to dogmatic discourse.

Our results are also consistent with the Uncertainty Management Model (van den Bos et al., 2005). Research within this framework suggests that it is not necessarily the existential threat that increases participants' worldview defense in such cases; rather, according to this perspective, the uncertainty about one's future, evoked by MS (e.g., when will I die? How will I die? Is there life after death?) is sometimes sufficient to give rise to compensatory processes that seek to restore a sense of certainty. Given that the current study analyzes real-world phenomena that occur outside the lab, we are limited in our ability to arbitrate between a TMT and an uncertainty management interpretation of the results.

According to TMT and the General Process Model of Threat and Defense (Jonas et al., 2014; Pyszczynski et al., 1999) people cope with threats by relying on both "distal" and "proximal” defense strategies. Proximal defenses (e.g., safety-related behavior, sense of anxiety) are activated when thoughts of death are at the focus of one's attention and entail a conscious processing by the experiencer. Distal defenses (e.g., worldview defense) on the other hand, 
operate when the threat is not in the focus of attention, do not involve conscious awareness and deliberation, and do not co-occur with proximal defense responses.

In our study, we saw that threats gave rise to increases in expressions of certainty (i.e., a distal defense) but also increased anxiety levels (i.e., a proximal reaction)—which seems inconsistent with TMT findings. Nevertheless, according to more recent research into the "fear+buffer perspective" (Lambert et al., 2014), MS manipulations actually often do generate an explicit sense of threat alongside distal defensive reactions. Therefore, the observed association between anxiety and certainty seems neither consistent nor inconsistent with the MS literature. More importantly, this association seems inconsistent with ATF which argues that anxiety and fear should cause a decrease in certainty expressions.

\section{The Language of Certainty}

The current findings join previous research showing that psychological distress causes people to become entrenched in their beliefs. For example, Randles et al. (2017) have shown that adverse life events (including existential threats such as illness and bereavement) cause people to endorse more extreme attitudes on a variety of topics, even years after the event. Additionally, much of the words that drive the certainty effect we observed herein (e.g., absolutely, everyone, all, nothing, must and never), i.e., absolutist words, were shown in previous research to be highly predictive of trait-level anxiety (Al-Mosaiwi \& Johnstone, 2018).

Expressions of certainty are about generating a picture of the world where one's proposition is a general, undeniable truth (rather than being contingent and probabilistic). They can be broken down to the scope of the proposition itself, and a propositional attitude. Consider the assertion "People love me. And you know what? I've been very successful. Everybody loves 
me" ${ }^{3}$. The universal quantifier "everybody" expands the possible worlds wherein the speaker is loved and packs the assertion with greater sense of certainty. Similarly, the statement "I am certain that she loves me" expresses an attitude of certainty towards the proposition (i.e., a propositional attitude) that "she loves me". Thus, certainty can be bolstered by using each of these two linguistic devices.

The effect of existential threats on certainty-related language was especially evident in quantification language, namely, in the words that denote the scope of one's assertion.

Following events of existential threats, participants utilized more universal quantifiers (e.g., all, everyone, nothing, must) — that generate blanket statements that do not leave room for much nuance or moderation.

In other words, the effect of threats seems to be more pronounced in linguistic devices that extend the scope of a proposition (i.e., universal quantification) rather than the attitudinal aspects towards the proposition. Some (but not all) of the words that denote attitudinal certainty (e.g., clearly) were actually slightly less pronounced under threat (albeit, not necessarily significantly so).

While, as noted, propositional attitudes express certainty, they also highlight the subjectivity of the proposition. For example, by saying "I am absolutely one hundred percent sure that she likes me" one is implicitly acknowledging that this is a belief, rather than a brute fact. In light of this, increasing the scope of a proposition (i.e., "everybody likes me") is a

\footnotetext{
${ }^{3}$ Donald Trump on CNN, July 2015; http://transcripts.cnn.com/TRANSCRIPTS/1507/08/acd.01.html
} 
potentially more effective means of arguing for the factivity of a belief, disallowing plurality of views or any subjectivity on the matter.

\section{Potential Implications}

The existential threats reviewed herein (COVID-19, acts of mass violence) were a hotbed to many conspiracy theories (van Prooijen, 2020). Theorizing within conspiracy research is consistent with the idea that people respond to such threatening events by relying on compensatory mechanisms; specifically, conspiracy research suggests that individuals cope with the existential anxiety and uncertainty associated with societal threats by strongly attaching themselves to conspiratorial beliefs that allow them to regain some sense of epistemic control (Douglas et al., 2017). Indeed, in a recent study focusing on an online community devoted to conspiracy theories, it was shown that following mass shootings and other dramatic events (e.g., the disappearance of the Malaysia Airlines flight MH370), conspiracy-related discourse became (even) more dogmatic and certain (Samory \& Mitra, 2018).

While being certain of one's beliefs may be psychologically comforting and sometimes useful (Bromberg-Martin \& Hikosaka, 2009), there are many downsides to being overly confident and dogmatic (Neale \& Bazerman, 1985). It is important for societies to be cognizant of these possible effects of enhanced exposure to existential threats on people's need for certainty - if we wish to handle the response to such threats in a constructive and reasoned manner.

\section{Potential Limitations and Future Directions}

In the current study, we measured participants' linguistic expression of certainty, rather than self-reports of participants' subjective sense of certainty; as such, our findings should be 
taken as evidence of change in the certitude of discourse, rather than of change in phenomenology. However, research on gauging psychological processes through text analysis reveals that unobtrusive language use is often a good indicator of subjective experience (Fan et al., 2019). Moreover, expressions on social media can be predictive of tangible disorders (e.g., depression (Eichstaedt et al., 2018) and consequential actions (e.g., suicide attempts; Gamoran et al., 2021), highlighting the potentially strong association between social media language and actual experience.

Our approach was based upon word counts calculated by pre-made dictionaries (namely, LIWC; Pennebaker et al., 2015). One well-known limitation of the word count approach is the insensitivity of the method to context (Iliev et al., 2015). For example, a tweet containing the phrase "I am not certain" would score high on certainty, because the word "certain" appears in it. However, the large datasets that usually accompany such analyses (including in the current studies) compensate for the likely random measurement error.

Another limitation of the current work is in our use of a quasi-experimental approach, building on ecological social media data. While such natural experiments offer new ways of psychological inquiry in the age of big data, quasi-experimental methods are still limited in their ability to support causal inferences (Shadish et al., 2002). Nonetheless, the use of big data methodology addressed core problems in social psychological research, namely, limitations of statistical power and ecological validity.

The societal discourse in which we are immersed is part and parcel of our mental lives (e.g., Mead, 1934); as such, the finding that existential threats increase the certainty of societal discourse is psychologically meaningful. However, it should not be assumed that societal 
discourse (or, more specifically, social media discourse) is a representative aggregate of the psychological states of the individual members of society.

First, it should be noted that despite its broad adoption, Twitter is not representative of the general population (Mellon \& Prosser, 2017; but see Kirilenko et al., 2017 for evidence that it is more diverse than typical study populations such as undergraduate students or MTurk workers). Furthermore, even within the population of Twitter users, individuals who feel uncertain might feel less of an urge to communicate this on social media; in contrast, individuals who decide to cope with threats in an active way might use Twitter to communicate messages of certainty. Thus, future research may benefit from examining expressions of certainty (or lack thereof) using different large-scale measures (for example, periodic surveys of people's worldviews and the extent to which they are confidently held); such designs could help us better characterize potentially divergent reactions of the different members of society.

It should also be noted that our sample consists of users solely from the United States and the United Kingdom, and therefore our results are potentially restricted to English speaking, western cultures. Future research could examine whether dynamics such as those observed herein are also found in non-English speaking countries, and in additional avenues wherein public conversations take place.

The current research is an example of work done in the context of "discovery" (Schindler et al., 2021). We did not conduct this research with the aim of supporting one theory or another, or with the aim of finding a significant difference in one direction or another. It could be argued that results that emerge from exploratory research are less convincing than results obtained from research that premeditatedly tests a clear directional prediction, explicated in a pre-registration. However, it is important to note that the studies herein rely on more than 2.5 million 
observations. Such a large sample size almost approximates the population itself (rather than being a sample). A main advantage of exploratory big-data research with such high evidential value is that even null findings can be compelling and of interest (see Simchon et al., 2020). Indeed, our findings were completely robust to different analytical choices and survived stringent multiple comparison correction (e.g., the $\mathrm{p}$-value for the main finding is $p<.00000001$ ).

Our findings join a large body of research in computational social science that explores real-world events via social media. When phenomena are studied through natural language processing, it is often the case that effect sizes are quite small (Boyd \& Schwartz, 2020; Iliev et al., 2015), and the utility of such small effects is often criticized. However, it is important to note that effects that are applicable to huge populations can be highly impactful, even if they are tiny (Funder \& Ozer, 2019).

For example, our estimate is that major mass shootings were associated with an increase of $21 \%$ in certainty-language (IRR $=1.21, p<.001)$. Nonetheless, this (relatively) small effect should be viewed in the context of multiplied exposure. In our study, we examined state-level effects for each mass shooting. The share of active twitter users in the United States stands at $22 \%$ (Pew Research Center, 2019). Thus, a small effect of $21 \%$ increase in certainty, multiplied by $22 \%$ of each state's population, could potentially have a huge impact on the nature of debate and discourse in a society. Indeed, analyses of longer trends show that there are major shifts in societal discourse that are highly consequential to democracy (e.g., Rathje et al., 2021; Stewart et al., 2019). Thus, when thinking about the interplay between the individual and the collective, it is important to note that small effects that impact the collective can lead us to a "death by a thousand needles." 


\section{Conclusion}

While our world is (arguably) safer than ever, we are increasingly exposed to representations of existential dangers. Such an environment may provide a hotbed for conspiracy theories and authoritarian leaders that fulfill individuals' epistemic needs by generating a false sense of certainty (Jost et al., 2007; Orehek et al., 2010). Future research may be able to delve deeper into the mechanisms wherein existential threats hurt individuals' capacity to freely and logically reason about the complexities of public life in a time of salient existential anxieties.

\section{Acknowledgments}

We wish to thank Dr. Jonathan Rosenblatt, Dr. Eran Bar-Kalifa and Civiqs.

\section{Author Contributions}

Conceptualization, A.S., M.G.; Data curation, A.S., C.T., M.G., T.S., I.K, and M.D.; Formal analysis, A.S.; Funding acquisition, M.G.; Investigation, A.S. and M.G.; Methodology, A.S. and M.G; Software, A.S. and C.T.; Supervision, M.G.; Validation, A.S..; Visualization, A.S.; Writing - review \& editing, A.S., and M.G.

\section{Declaration of Conflicting Interests}

Authors declare no competing interests

\section{Funding}

United States - Israel Binational Science Foundation grant no. 2015258 to M.G; Israel Science Foundation grant no. $1113 / 18$ to M.G 


\section{Open Practices}

All the data and code are available at

https://osf.io/9tfyw/?view_only=124efbecf88a426e8ab8a4417db7e330 and will be made public upon acceptance.

\section{References}

Al-Mosaiwi, M., \& Johnstone, T. (2018). In an Absolute State: Elevated Use of Absolutist Words Is a Marker Specific to Anxiety, Depression, and Suicidal Ideation. Clinical Psychological Science, 6(4), 529-542.

Arndt, J., Greenberg, J., Pyszczynski, T., \& Solomon, S. (1997). Subliminal Exposure to DeathRelated Stimuli Increases Defense of the Cultural Worldview. Psychological Science, 8(5), $379-385$.

Betancourt, H., \& Blair, I. (1992). A cognition (attribution)-emotion model of violence in conflict situations. Personality \& Social Psychology Bulletin, 18(3), 343-350.

Boyd, R. L., \& Schwartz, H. A. (2020). Natural Language Analysis and the Psychology of Verbal Behavior: The Past, Present, and Future States of the Field. Journal of Language and Social Psychology, 40(1), 21-41.

Bromberg-Martin, E. S., \& Hikosaka, O. (2009). Midbrain dopamine neurons signal preference for advance information about upcoming rewards. Neuron, 63(1), 119-126.

Brück, T., \& Müller, C. (2010). Comparing the determinants of concern about terrorism and crime. Global Crime, 11(1), 1-15.

Brysbaert, M., \& Stevens, M. (2018). Power analysis and effect size in mixed effects models: A tutorial. Journal of cognition, 1(1), 9. 
Bullock, J. G., Green, D. P., \& Ha, S. E. (2010). Yes, but what's the mechanism?(don't expect an easy answer). Journal of Personality and Social Psychology, 98(4), 550.

Canetti-Nisim, D., Halperin, E., Sharvit, K., \& Hobfoll, S. E. (2009). A New Stress-Based Model of Political Extremism: Personal Exposure to Terrorism, Psychological Distress, and Exclusionist Political Attitudes. The Journal of Conflict Resolution, 53(2), 363-389.

Chatard, A., Hirschberger, G., \& Pyszczynski, T. (2020). A word of caution about many labs 4: If you fail to follow your preregistered plan, you may fail to find a real effect. https://psyarxiv.com/ejubn/download?format=pdf

Cohen, S. J. (2012). Construction and preliminary validation of a dictionary for cognitive rigidity: linguistic markers of overconfidence and overgeneralization and their concomitant psychological distress. Journal of Psycholinguistic Research, 41(5), 347-370.

Corley, P. C., \& Wedeking, J. (2014). The (Dis)Advantage of Certainty: The Importance of Certainty in Language. Law \& Society Review, 48(1), 35-62.

Cox, C. R., \& Kersten, M. (2016). Mortality salience increases language style matching and well-being. Self and Identity: The Journal of the International Society for Self and Identity, $15(4), 452-467$.

Dong, E., Du, H., \& Gardner, L. (2020). An interactive web-based dashboard to track COVID-19 in real time. The Lancet Infectious Diseases, 20(5), 533-534.

Dore, B., Ort, L., Braverman, O., \& Ochsner, K. N. (2015). Sadness Shifts to Anxiety Over Time and Distance From the National Tragedy in Newtown, Connecticut. Psychological Science, 26(4), 363-373.

Douglas, K. M., Sutton, R. M., \& Cichocka, A. (2017). The Psychology of Conspiracy Theories. Current Directions in Psychological Science, 26(6), 538-542. 
Eichstaedt, J. C., Smith, R. J., Merchant, R. M., Ungar, L. H., Crutchley, P., Preoţiuc-Pietro, D., Asch, D. A., \& Schwartz, H. A. (2018). Facebook language predicts depression in medical records. Proceedings of the National Academy of Sciences of the United States of America, 115(44), 11203-11208.

Ellsworth, P. C., \& Scherer, K. R. (2003). Appraisal processes in emotion. Handbook of Affective Sciences, 572, V595.

Fan, R., Varol, O., Varamesh, A., Barron, A., van de Leemput, I. A., Scheffer, M., \& Bollen, J. (2019). The minute-scale dynamics of online emotions reveal the effects of affect labeling. Nature Human Behaviour, 3(1), 92-100.

Fast, E., \& Horvitz, E. (2016). Identifying Dogmatism in Social Media: Signals and Models. In arXiv [cs.CL]. arXiv. http://arxiv.org/abs/1609.00425

Fritsche, I., Cohrs, J. C., Kessler, T., \& Bauer, J. (2012). Global warming is breeding social conflict: The subtle impact of climate change threat on authoritarian tendencies. Journal of Environmental Psychology, 32(1), 1-10.

Funder, D. C., \& Ozer, D. J. (2019). Evaluating Effect Size in Psychological Research: Sense and Nonsense. Advances in Methods and Practices in Psychological Science, 2(2), 156-168.

Gamoran, A., Kaplan, Y., Orr, R. I., Simchon, A., \& Gilead, M. (2021). Using PsychologicallyInformed Priors for Suicide Prediction in the CLPsych 2021 Shared Task. Proceedings of the Seventh Workshop on Computational Linguistics and Clinical Psychology: Improving Access, 103-109.

Garcia, D., \& Rimé, B. (2019). Collective Emotions and Social Resilience in the Digital Traces After a Terrorist Attack. Psychological Science, 30(4), 617-628.

Gentry, J. (2012). twitteR: R based Twitter client. https://cran.r- 
project.org/web/packages/twitteR/twitteR.pdf

Greenberg, J., Pyszczynski, T., \& Solomon, S. (1986). The Causes and Consequences of a Need for Self-Esteem: A Terror Management Theory. In R. F. Baumeister (Ed.), Public Self and Private Self (pp. 189-212). Springer New York.

Grosz, M. P., Rohrer, J. M., \& Thoemmes, F. (2020). The Taboo Against Explicit Causal Inference in Nonexperimental Psychology. Perspectives on Psychological Science: A Journal of the Association for Psychological Science, 15(5), 1243-1255.

Hardin, C. D., \& Higgins, E. T. (1996). Shared reality: How social verification makes the subjective objective. In R. M. Sorrentino (Ed.), Handbook of motivation and cognition, Vol (Vol. 3, pp. 28-84). The Guilford Press, xxvi.

Hays, J. N. (2005). Epidemics and pandemics: their impacts on human history. Abc-clio.

Heine, S. J., Proulx, T., \& Vohs, K. D. (2006). The meaning maintenance model: on the coherence of social motivations. Personality and Social Psychology Review: An Official Journal of the Society for Personality and Social Psychology, Inc, 10(2), 88-110.

Iliev, R., Dehghani, M., \& Sagi, E. (2015). Automated text analysis in psychology: Methods, applications, and future developments. Language and Cognition, 7(2), 265-290.

Jonas, E., McGregor, I., Klackl, J., Agroskin, D., Fritsche, I., Holbrook, C., Nash, K., Proulx, T., \& Quirin, M. (2014). Threat and Defense: From Anxiety to Approach. In J. M. Olson \& M. P. Zanna (Eds.), Advances in Experimental Social Psychology (Vol. 49, pp. 219-286). Academic Press.

Jonas, E., Schimel, J., Greenberg, J., \& Pyszczynski, T. (2002). The Scrooge Effect: Evidence that Mortality Salience Increases Prosocial Attitudes and Behavior. Personality \& Social Psychology Bulletin, 28(10), 1342-1353. 
Jong, J., Halberstadt, J., \& Bluemke, M. (2012). Foxhole atheism, revisited: The effects of mortality salience on explicit and implicit religious belief. Journal of Experimental Social Psychology, 48(5), 983-989.

Jost, J. T., Napier, J. L., Thorisdottir, H., Gosling, S. D., Palfai, T. P., \& Ostafin, B. (2007). Are needs to manage uncertainty and threat associated with political conservatism or ideological extremity? Personality \& Social Psychology Bulletin, 33(7), 989-1007.

Kahn, J. H., Tobin, R. M., Massey, A. E., \& Anderson, J. A. (2007). Measuring emotional expression with the Linguistic Inquiry and Word Count. The American Journal of Psychology, 120(2), 263-286.

Kashdan, T. B., Nathan DeWall, C., Schurtz, D. R., Deckman, T., Lykins, E. L. B., Evans, D. R., McKenzie, J., Segerstrom, S. C., Gailliot, M. T., \& Brown, K. W. (2014). More than words: Contemplating death enhances positive emotional word use. Personality and Individual Differences, 71, 171-175.

Kennedy, B., Ashokkumar, A., Boyd, R. L., \& Dehghani, M. (2021). Text Analysis for Psychology: Methods, Principles, and Practices. https://doi.org/10.31234/osf.io/h2b8t

Kilbourne, E. D. (2006). Influenza pandemics of the 20th century. Emerging Infectious Diseases, 12(1), 9 .

Kirilenko, A. P., Desell, T., Kim, H., \& Stepchenkova, S. (2017). Crowdsourcing Analysis of Twitter Data on Climate Change: Paid Workers vs. Volunteers. Sustainability: Science Practice and Policy, 9(11), 2019.

Klein, R. A., Cook, C. L., Ebersole, C. R., Vitiello, C. A., Nosek, B. A., Chartier, C. R., Christopherson, C. D., Clay, S., Collisson, B., Crawford, J., \& al., E. (2019). Many Labs 4: Failure to Replicate Mortality Salience Effect With and Without Original Author 
Involvement. https://doi.org/10.31234/osf.io/vef2c

Kopietz, R., Hellmann, J. H., Higgins, E. T., \& Echterhoff, G. (2010). Shared-Reality Effects on Memory: Communicating to Fulfill Epistemic Needs. Social Cognition, 28(3), 353-378.

Lambert, A. J., Eadeh, F. R., Peak, S. A., Scherer, L. D., Schott, J. P., \& Slochower, J. M. (2014). Toward a greater understanding of the emotional dynamics of the mortality salience manipulation: Revisiting the "affect-free" claim of terror management research. Journal of Personality and Social Psychology, 106(5), 655-678.

Landau, M. J., Greenberg, J., Sullivan, D., Routledge, C., \& Arndt, J. (2009). The protective identity: Evidence that mortality salience heightens the clarity and coherence of the selfconcept. Journal of Experimental Social Psychology, 45(4), 796-807.

Lazarus, R. S. (1991). Progress on a cognitive-motivational-relational theory of emotion. The American Psychologist, 46(8), 819-834.

Leatherdale, S. T. (2019). Natural experiment methodology for research: a review of how different methods can support real-world research. International Journal of Social Research Methodology, 22(1), 19-35.

Lerner, J. S., Han, S., \& Keltner, D. (2007). Feelings and Consumer Decision Making: Extending the Appraisal-Tendency Framework. Journal of Consumer Psychology: The Official Journal of the Society for Consumer Psychology, 17(3), 181-187.

Lerner, J. S., \& Keltner, D. (2000). Beyond valence: Toward a model of emotion-specific influences on judgement and choice. Cognition and Emotion, 14(4), 473-493.

Lerner, J. S., \& Keltner, D. (2001). Fear, anger, and risk. Journal of Personality and Social Psychology, 81(1), 146-159.

Maguen, S., Papa, A., \& Litz, B. T. (2008). Coping with the threat of terrorism: a review. 
Anxiety, Stress, and Coping, 21(1), 15-35.

Mead, G. H. (1934). Mind, self and society. University of Chicago Press.

Mellon, J., \& Prosser, C. (2017). Twitter and Facebook are not representative of the general population: Political attitudes and demographics of British social media users. Research \& Politics, 4(3), 2053168017720008.

Motro, D., \& Sullivan, D. (2017). Could two negative emotions be a positive? The effects of anger and anxiety in enemyship. Journal of Experimental Social Psychology, 69, 130-143.

Neale, M. A., \& Bazerman, M. H. (1985). The Effects of Framing and Negotiator Overconfidence on Bargaining Behaviors and Outcomes. Academy of Management Journal, 28(1), 34-49.

Orehek, E., Fishman, S., Dechesne, M., Doosje, B., Kruglanski, A. W., Cole, A. P., Saddler, B., \& Jackson, T. (2010). Need for Closure and the Social Response to Terrorism. Basic and Applied Social Psychology, 32(4), 279-290.

Pashler, H., \& Wagenmakers, E.-J. (2012). Editors' Introduction to the Special Section on Replicability in Psychological Science: A Crisis of Confidence? Perspectives on Psychological Science: A Journal of the Association for Psychological Science, 7(6), 528530.

Pennebaker, J. W., Boyd, R. L., Jordan, K., \& Blackburn, K. (2015). The Development and Psychometric Properties of LIWC2015. https://repositories.lib.utexas.edu/bitstream/handle/2152/31333/LIWC2015_LanguageManu al.pdf

Pennebaker, J. W., Mayne, T. J., \& Francis, M. E. (1997). Linguistic predictors of adaptive bereavement. Journal of Personality and Social Psychology, 72(4), 863-871. arXiv. 
Pew Research Center. (2019, April 10). Share of U.S. adults using social media, including Facebook, is mostly unchanged since 2018. https://www.pewresearch.org/facttank/2019/04/10/share-of-u-s-adults-using-social-media-including-facebook-is-mostlyunchanged-since-2018/

Pezzuti, T., Leonhardt, J. M., \& Warren, C. (2021). Certainty in Language Increases Consumer Engagement on Social Media. Journal of Interactive Marketing, 53, 32-46.

Pinker, S. (2011). The better angels of our nature: Why violence has declined. New York, NY: Viking.

Pyszczynski, T., Abdollahi, A., Solomon, S., Greenberg, J., Cohen, F., \& Weise, D. (2006). Mortality salience, martyrdom, and military might: the great satan versus the axis of evil. Personality \& Social Psychology Bulletin, 32(4), 525-537.

Pyszczynski, T., Greenberg, J., \& Solomon, S. (1999). A dual-process model of defense against conscious and unconscious death-related thoughts: an extension of terror management theory. Psychological Review, 106(4), 835-845.

Pyszczynski, T., Lockett, M., Greenberg, J., \& Solomon, S. (2021). Terror Management Theory and the COVID-19 Pandemic. Journal of Humanistic Psychology, 61(2), 173-189.

Raghunathan, R., \& Pham, M. T. (1999). All Negative Moods Are Not Equal: Motivational Influences of Anxiety and Sadness on Decision Making. Organizational Behavior and Human Decision Processes, 79(1), 56-77.

Randles, D., Heine, S. J., Poulin, M., \& Silver, R. C. (2017). Experienced Adversity in Life Is Associated With Polarized and Affirmed Political Attitudes. Social Psychological and Personality Science, 8(6), 652-659.

Rathje, S., Van Bavel, J. J., \& van der Linden, S. (2021). Out-group animosity drives 
engagement on social media. Proceedings of the National Academy of Sciences of the United States of America, 118(26). https://doi.org/10.1073/pnas.2024292118

Ripley, B., Venables, B., Bates, D. M., Hornik, K., Gebhardt, A., Firth, D., \& Ripley, M. B. (2013). Package "mass." Cran R. https://cran.revolutionanalytics.com/web/packages/MASS/MASS.pdf

Rokeach, M. (1954). The nature and meaning of dogmatism. Psychological Review, 61(3), 194204.

Russell, P. S., \& Giner-Sorolla, R. (2011). Moral anger, but not moral disgust, responds to intentionality. Emotion, 11(2), 233-240.

Samory, M., \& Mitra, T. (2018). Conspiracies Online: User Discussions in a Conspiracy Community Following Dramatic Events. Proceedings of the International AAAI Conference on Web and Social Media, 12(1). https://ojs.aaai.org/index.php/ICWSM/article/view/15039

Schindler, S., Reinhardt, N., \& Reinhard, M. A. (2021). Defending one's worldview under mortality salience: Testing the validity of an established idea. Journal of Experimental Social Psychology, 93, 104087.

Schwartz, H. A., Eichstaedt, J. C., Kern, M. L., Dziurzynski, L., Ramones, S. M., Agrawal, M., Shah, A., Kosinski, M., Stillwell, D., Seligman, M. E. P., \& Ungar, L. H. (2013). Personality, gender, and age in the language of social media: the open-vocabulary approach. PloS One, 8(9), e73791.

Sell, A., Tooby, J., \& Cosmides, L. (2009). Formidability and the logic of human anger. Proceedings of the National Academy of Sciences of the United States of America, 106(35), 15073-15078.

Shadish, W. R., Cook, T. D., \& Campbell, D. T. (2002). Quasi-experimental designs that either 
lack a control group or lack pretest observations on the outcome. Experimental and QuasiExperimental Designs for Generalized Causal Inference, 103-134.

Shields, C. G., Finley, M. A., Elias, C. M., Coker, C. J., Griggs, J. J., Fiscella, K., \& Epstein, R. M. (2013). Pain assessment: the roles of physician certainty and curiosity. Health Communication, 28(7), 740-746.

Slone, M., \& Shoshani, A. (2006). Evaluation of preparatory measures for coping with anxiety raised by media coverage of terrorism. Journal of Counseling Psychology, 53(4), 535.

Smith, C. A., \& Ellsworth, P. C. (1985). Patterns of cognitive appraisal in emotion. Journal of Personality and Social Psychology, 48(4), 813-838.

Sterling, J., Jost, J. T., \& Bonneau, R. (2020). Political psycholinguistics: A comprehensive analysis of the language habits of liberal and conservative social media users. Journal of Personality and Social Psychology, 118(4), 805-834.

Stewart, A. J., Mosleh, M., Diakonova, M., Arechar, A. A., Rand, D. G., \& Plotkin, J. B. (2019). Information gerrymandering and undemocratic decisions. Nature, 573(7772), 117-121.

Tamir, M., Mitchell, C., \& Gross, J. J. (2008). Hedonic and instrumental motives in anger regulation. Psychological Science, 19(4), 324-328.

Tingley, D., Yamamoto, T., Hirose, K., Keele, L., \& Imai, K. (2014). mediation: R package for causal mediation analysis. https://dspace.mit.edu/handle/1721.1/91154?show=full van den Bos, K., \& Lind, E. A. (2002). Uncertainty management by means of fairness judgments. In M. P. Zanna (Ed.), Advances in experimental social psychology, Vol (Vol. 34, pp. 1-60). Academic Press, x.

van den Bos, K., Poortvliet, P. M., Maas, M., Miedema, J., \& van den Ham, E.-J. (2005). An enquiry concerning the principles of cultural norms and values: The impact of uncertainty 
and mortality salience on reactions to violations and bolstering of cultural worldviews. Journal of Experimental Social Psychology, 41(2), 91-113.

van Prooijen, J.-W. (2020). An Existential Threat Model of Conspiracy Theories. European Psychologist, 25(1), 16-25.

van Prooijen, J.-W., \& Krouwel, A. P. M. (2017). Extreme Political Beliefs Predict Dogmatic Intolerance. Social Psychological and Personality Science, 8(3), 292-300.

Verdone, M. (2014). Python twitter tools.

Wang, C., Pan, R., Wan, X., Tan, Y., Xu, L., \& Ho, C. S. (2020). Immediate psychological responses and associated factors during the initial stage of the 2019 coronavirus disease (COVID-19) epidemic among the general .... International Journal of. https://www.mdpi.com/1660-4601/17/5/1729

Yarkoni, T. (2020). The generalizability crisis. The Behavioral and Brain Sciences, 1-37. 
